\title{
567.
}

\section{ON AN IDENTICAL EQUATION CONNECTED WITH THE THEORY OF INVARIANTS.}

[From the Quarterly Journal of Pure and Applied Mathematics, vol. XII. (1873), pp. 115-118.]

WRITE

$$
\begin{aligned}
& a=g-h, \\
& b=h-f, \\
& c=f-g,
\end{aligned}
$$

equations implying a fourth equation forming with them the system

$$
\begin{array}{r}
-h+g-a=0 \\
h-f-b=0 \\
-g+f \cdot-c=0 \\
a+b+c \quad=0
\end{array}
$$

and also

Then, putting for shortness

$$
a f+b g+c h=0 .
$$

$$
\begin{aligned}
& P=(b g-c h)(c h-a f)(a f-b g), \\
& Q=a^{2} g^{2} h^{2}+b^{2} h^{2} f^{2}+c^{2} f^{2} g^{2}+a^{2} b^{2} c^{2}, \\
& R=a^{2} f^{2}\left(a^{2}+f^{2}\right)+b^{2} g^{2}\left(b^{2}+g^{2}\right)+c^{2} h^{2}\left(c^{2}+h^{2}\right),
\end{aligned}
$$

we have

$$
2 P+Q-R=0,
$$

viz. substituting for $a, b, c$ their values $g-h, h-f, f-g$, this is an identical equation. 
567] AN IDENTICAL EQUATION CONNECTED WITH THE THEORY OF INVARIANTS. 53

The direct verification is however somewhat tedious, and the equation may be proved more easily as follows: find

In the terms $a^{2}+f^{2}, b^{2}+g^{2}, c^{2}+h^{2}$ of $R$, substituting for $a, b, c$ their values, we

$$
\begin{gathered}
R=\left(f^{2}+g^{2}+h^{2}\right)\left(a^{2} f^{2}+b^{2} g^{2}+c^{2} h^{2}\right) \\
-2 f g h\left(a^{2} f+b^{2} g+c^{2} h\right),
\end{gathered}
$$

which may be written

$$
\begin{aligned}
R= & -2\left(f^{2}+g^{2}+h^{2}\right)(b c g h+c a h f+a b f g) \\
& -2 f g h\left(a^{2} f+b^{2} g+c^{2} h\right) .
\end{aligned}
$$

We have then

$$
2 P=-2 b c g h(b g-c h)-2 c a h f(c h-a f)-2 a b f g(a f-b g),
$$

and thence

$$
\begin{aligned}
2 P-R & =2 b c g h\left(f^{2}+g^{2}+h^{2}-b g+c h\right) \\
& +2 c a h f\left(f^{2}+g^{2}+h^{2}-c h+a f\right) \\
& +2 a b f g\left(f^{2}+g^{2}+h^{2}-a f+b g\right) \\
& +2 f g h\left(a^{2} f+b^{2} g+c^{2} h\right),
\end{aligned}
$$

which is at once converted into

$$
\begin{aligned}
2 P-R & =2 b c g h\left\{a^{2}+f(f+g+h)\right\} \\
& +2 c a h f\left\{b^{2}+g(f+g+h)\right\} \\
& +2 a b f g\left\{c^{2}+h(f+g+h)\right\} \\
& +2 f g h\left(a^{2} f+b^{2} g+c^{2} h\right)
\end{aligned}
$$

or, what is the same thing,

$$
2 P-R=2 f g h\left\{(b c+c a+a b)(f+g+h)+a^{2} f+b^{2} g+c^{2} h\right\}+2 a b c(a g h+b h f+c f g),
$$

where, since

the last term is

$$
a g h+b h f+c f g=-a b c
$$

$$
=-2 a^{2} b^{2} c^{2}
$$

But from the equation last written down we deduce at once

and we thence have

$$
Q=2 a^{2} b^{2} c^{2}-2 f g h(b c f+c a g+a b h)
$$

$$
2 P+Q-R=2 f g h\left\{(b c+c a+a b)(f+g+h)+\left(a^{2} f+b^{2} g+c^{2} h\right)-b c f-c a g-a b h\right\},
$$

which is

$$
=2 f g h(a+b+c)(a f+b g+c h),
$$

and consequently $=0$, the theorem in question. 
54 AN IDENTICAL EQUATION CONNECTED WITH THE THEORY OF INVARIANTS. [567

Instead of $a, b, c, f, g, h, \mathrm{I}$ write $a W \div Y Z, b W \div Z X, c W \div X Y, f \div X, g \div Y, h \div Z$ : we have therefore

and as before

$$
\begin{array}{r}
-h Y+g Z-a W=0 \\
h X \cdot-f Z-b W=0 \\
-g X+f Y \cdot-c W=0 \\
a X+b Y+c Z \cdot \quad=0
\end{array}
$$

$$
a f+b g+c h=0 \text {. }
$$

Moreover, omitting a common factor, the new values of $P, Q, R$ are

$$
\begin{aligned}
& P=X Y Z W(b g-c h)(c h-a f)(a f-b g), \\
& Q=a^{2} g^{2} h^{2} X^{4}+b^{2} h^{2} f^{2} Y^{4}+c^{2} f^{2} g^{2} Z^{4}+a^{2} b^{2} c^{2} W^{4}, \\
& R=a^{2} f^{2}\left(a^{2} X^{2} W^{2}+f^{2} Y^{2} Z^{2}\right)+b^{2} g^{2}\left(b^{2} Y^{2} W^{2}+g^{2} Z^{2} X^{2}\right)+c^{2} h^{2}\left(c^{2} Z^{2} W^{2}+h^{2} X^{2} Y^{2}\right),
\end{aligned}
$$

and the identical equation is, as before,

$$
2 P+Q-R=0
$$

Consider the operative symbols

$$
\begin{aligned}
& d_{x_{1}}, d_{x_{2}}, d_{x_{3}}, d_{x_{4}}, \\
& d_{y_{1}}, d_{y_{2}}, d_{y_{3}}, d_{y_{4}},
\end{aligned}
$$

and write $a=d_{x_{1}} d_{y_{2}}-d_{y_{1}} d_{x_{2}}=12$, \&c., that is

$$
\begin{array}{ll}
a=23, & f=14, \\
b=31, & g=24, \\
c=12, & h=34,
\end{array}
$$

and also $X=x d_{x_{1}}+y d_{y_{1}}$, \&c. say

$$
X=\nabla_{1}, \quad Y=\nabla_{2}, \quad Z=\nabla_{3}, \quad W=\nabla_{4} .
$$

These values of $a, b, c, f, g, h, X, Y, Z, W$ satisfy the above written equations of connexion, and therefore the identical equation $2 P+Q-R=0$. Hence taking $U$ to denote the quartic function $U=(a, b, c, d, e)(x, y)^{4}$, and therefore $U_{1}=(a, \ldots)\left(x_{1}, y_{1}\right)^{4}, \& c$., we have

$$
(2 P+Q-R) U_{1} U_{2} U_{3} U_{4}=0
$$

where, after the differentiations, $\left(x_{1}, y_{1}\right), \ldots,\left(x_{4}, y_{4}\right)$ are to be each of them replaced by $(x, y)$.

Observe that $P$ is the sum of three positive and three negative terms, but that after the omission of the suffixes each term taken with its proper sign becomes equal to the same quantity, and the value of $P$ is $=6$ times any one term thereof. Thus omitting for the moment the factor $\nabla_{1} \nabla_{2} \nabla_{3} \nabla_{4}$, two of the terms are $-(a f)^{2} b g+a f(b g)^{2}$, that is,

$$
-(14.23)^{2}(24.31)+(14.23)(24.31)^{2}
$$


567] AN IDENTICAL EQUATION CONNECTED WITH THE THEORY OF INVARIANTS. 55

and, if in the first term we interchange 3 and 4 , it becomes $-(13.24)^{2}(23.41)$, that is, $+(14.23)(24.31)^{2}$, viz. it becomes equal to the second term. As regards $Q$ the terms are all positive and become equal to each other; and the like as regards $R$ : hence we have

$$
\left\{12 \nabla_{1} \nabla_{2} \nabla_{3} \nabla_{4}(14.23)(24.31)^{2}+4 \nabla_{1}^{4}(23)^{2}(34)^{2}(42)^{2}-6 \nabla_{1}{ }^{2} \nabla_{4}{ }^{2}(43)^{4}(14)^{2}\right\} U_{1} U_{2} U_{3} U_{4}=0 \text {, }
$$

which, omitting a numerical factor $6 \cdot 2 \cdot 12^{2} \cdot 2 \cdot 24^{2} \cdot 4,=3^{5} \cdot 2^{15}$, is in fact the well-known equation

where

$$
\Omega+J U-I H=0,
$$

$$
\begin{aligned}
& U=(a, b, c, d, e)(x, y)^{4}, \\
\Omega & =\operatorname{disct.}(a x+b y, b x+c y, c x+d y, d x+e y)(\xi, \eta)^{3} \\
= & (a x+b y)^{2}(d x+e y)^{2}+\& c . \\
I & =a e-4 b d+3 c^{2}, \\
J & =a c e-a d^{2}-b^{2} c-c^{3}+2 b c d,
\end{aligned}
$$

viz. attending only to the coefficient of $x^{4}$, this equation is

$a^{2} d^{2}+4 a c^{3}+4 b^{3} d-3 b^{2} c^{2}-6 a b c d+a\left(a c e-a d^{2}-b^{2} e-c^{3}+2 b c d\right)+\left(a c-b^{2}\right)\left(a e-4 b d+3 c^{2}\right)=0$. 